(C) 2018 IEEE. Personal use of this material is permitted. Permission from IEEE must be obtained for all other uses, in any current or future media, including reprinting/republishing this material for advertising or promotional purposes, creating new collective works, for resale or redistribution to servers or lists, or reuse of any copyrighted component of this work in other works. 


\title{
Feature Selection for the Detection of Sleep Apnea using Multi-Bio Signals from Overnight Polysomnography*
}

\author{
Xilin $\mathrm{Li}^{1}$ and Ahmed Al-Ani ${ }^{2}$ and Sai Ho Ling ${ }^{3}$
}

\begin{abstract}
Patients with sleep apnea (SA) are at increased risk of stroke and cardiovascular disease. Diagnosis of sleep apnea depends on the standard overnight polysomnography (PSG). In this study, the DREAM Apnea Database was used to evaluate the importance of the various features proposed in the literature for the analysis of sleep apnea. Various timeand frequency- domain features that include wavelet and power spectral density were extracted from ECG, EMG, EEG, airflow, $\mathrm{SaO2}$, abdominal and thoracic recordings. Evaluation measures of one-way analysis of variance (ANOVA) and Rank-Sum test were used to test the performance of different features. The selected feature subset indicated that frequency-domain features outperform time-domain ones. This study will help in enhancing the detection accuracy of sleep apnea for the various polysomnography signals.
\end{abstract}

\section{INTRODUCTION}

There are three basic types of respiratory disturbances in sleeping, and the most common sleep disorder is sleep apnea. Sleep apnea (SA) is considered as a temporary closure of the upper airway repeatedly during sleep and it leads to the complete cessation of breathing for more than 10 seconds in adults [1]. Sleep apnea is found to be associated with increased mortality, stroke and cardiovascular disease. Sleep apnea is classified into three types, Obstructive Sleep Apnea (OSA), Central Sleep Apnea (CSA) and Mixed Sleep Apnea (MSA). To exacerbate SA, it has been recommended that SA should be objectively evaluated. The ApneaHypopnea Index (AHI) is an index used to indicate the severity of sleep apnea. It is represented by the number of apnea and hypopnea events per hour of sleep. In adults, an AHI that is more than five or a minimum oxygen saturation of less than 85 percent is defined abnormal [2]. The gold standard for diagnosing sleep apnea is overnight polysomnography (PSG, see in Figure 1). This diagnosis includes the monitoring of the breath airflow, snore, midsagittal jaw movement, respiratory events, oxygen saturation $(\mathrm{SaO} 2)$, body position, electroencephalography (EEG), electromyography (EMG), electrooculography (EOG), and electrocardiography (ECG) [3]. Since the PSG recording lasts the whole night and the data of PSG is huge, the detection by expert physicians is a

*This work was not supported by any organization

${ }^{1}$ Xilin $\mathrm{Li}$ is with the School of Biomedical Engineering, Faculty of Engineering and Information Technology (FEIT), University of Technology Sydney (UTS), NSW 2007, Australia Xilin.Li@student.uts.edu.au

${ }^{2}$ Ahmed Al-Ani is with the School of Biomedical Engineering, Faculty of Engineering and Information Technology (FEIT), University of Technology Sydney (UTS), NSW 2007, Australia Ahmed. Al-Ani@uts . edu . au

${ }^{3}$ Sai Ho Ling is with the School of Biomedical Engineering, Faculty of Engineering and Information Technology (FEIT), University of Technology Sydney (UTS), NSW 2007, Australia Steve. Ling@uts. edu. au time-consuming task. Thus, automatic detection is desirable. In automatic detection, feature extraction and selection play an important role. Several pervious studies attempted to achieve the goal of obtaining better feature sets. Most of the existing feature evaluation studies focus on limited number of PSG signals, where the efficacy of the selected feature set is evaluated based on the classification performance.

In general, sleep respiratory disturbances are related to desaturation. Oxygen saturation $(\mathrm{SaO} 2)$ and airflow are usually utilized to detect apnea. For example, three methods were applied to extract features from oxygen saturation signals in [4]: approximate entropy (ApEn), central tendency measure (CTM) and Lempel-Ziv Complexity (LZC). Multilayer perceptron network provided a diagnosis when fed with those input features. Otero et al. showed the relationship between apnea and the drop in oxygen saturation [5]. The SA diagnosis algorithm was based on a computational representation of the morphological criteria from a physician, and a Multivariable Fuzzy Temporal Profile model. Gunes et al. [6] used four features and a multi-layer perceptron artificial neural network to identify sleep apnea. These features included arousal index, apnea and hypopnea index, the $\mathrm{SaO} 2$ minimum value in the stage of rapid eye movement and percent sleep time. The level-5-detail coefficients were obtained by Discrete Wavelet Transformation and used as the inputs for a feedforward artificial neural network [7]. In a recent work by Otero et al., it was reported that sudden changes in the basal value of the respiratory airflow was found in apneic polysomnography fragments, and the amount of inhaled and exhaled air. A fuzzy set was used for the apnea identification [8].

Sleep respiratory disturbances are accompanied by changes in electrocardiography (ECG), electroencephalography (EEG) and chin Electromyography (EMG). Therefore, there is an increased interest by researchers to utilize these signals. In [9], a heuristic splitting and relevance approach was proposed to extract features from ECG signals and classify those features using a K-nearest neighbor classifier. Hassan et al. [10] calculated statistical and spectral features from the ECG signals to differentiate between apneic and non-apneic conditions. Bootstrap aggregation was used to classify apneic portions of data from normal ones. Bandpass filter and Hilbert-Huang Transformation were utilized to calculate trend features [11]. Ubeyli et al. [12] presented an adaptive neuro-fuzzy inference system (ANFIS) to diagnose SA using EEG signals. This method was performed in two stages: feature extraction using Discrete Wavelet Transformation and classification by the ANFIS. Other studies 
Fig. 1. Example of Polysomnography signal

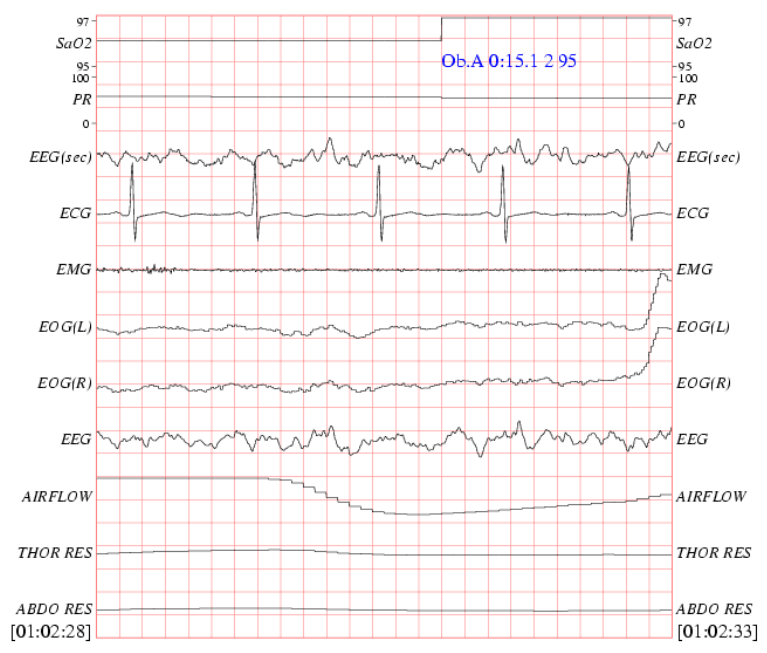

considered chin EMG, as muscle activities was found to play an important role in identifying apnea portions [13], [14], [15]. Some other studies focused on abdominal and thoracic signals since there is a correlation between two-effort signals with the patients airflow signal. In [16], the two-effort signals were decomposed into spectral components using Discrete Wavelet Transformation and the mean energy levels were used as inputs for multiplayer neural networks. Similarly, the abdominal respiratory signals were decomposed by Discrete Wavelet Transformation and the spectral components were used to train an artificial neural network [17].

In this paper, we proposed a new algorithm for evaluating a number of feature extraction methods that can be applied to analyse various polysomnography signals in terms of their capability in detecting sleep apnea. The one-way analysis of variance (ANOVA) and rank-sum test were used to evaluate the importance of features. The goal of this work is to build a robust and efficient feature set that can be used for detecting sleep apnea. The next section discusses the features that will be extracted from the different sleep apnea signals.

\section{MATERIAL AND METHODS}

The DREAM Apnea Database that consists of 12 PSG signals is used in this study. Each recording consists of ECG, EMG, EEG, airflow, SaO2, abdominal and thoracic signals, and lasts for approximately $9 \mathrm{~h}$. The PSG data are recorded at $200 \mathrm{~Hz}$ sampling frequency. These data recordings are annotated based on the respiratory events by an expert. Each signal is divided into $10 \mathrm{~s}$ windows since this duration is the minimum obstruction duration to detect an SA.

In the paper, we used wavelet transformation and power spectral density to extract frequency-domain features. Wavelet transformation is an extension of the classical Fourier transform. It works on a multi-scale basis and is suitable to analyze non-stationary signals because it has a varying window size, i.e., broad at low frequencies and narrow at high frequencies. Wavelet transformation employs scaling functions and wavelet functions, being related to low-pass and high-pass filters, to decompose signals into an approximation level and detail levels. For example, a wavelet of depth 9 provides a decomposition of a given signal into a set of the approximation coefficient (A9) and detail levels (i.e. D8). On the other hand, power spectral density can describe the distribution of frequency power components. Power spectral density estimation methods can be divided into non-parametric and parametric. The nonparametric methods have less computational complexity, while parametric methods are generally more accurate as they depend on some a priori knowledge to calculate the power spectral density.

\section{A. ECG features}

It has been stated in previous studies that the ECG signals of SA patients show distinct patterns in R-R intervals, with lower heart rates than healthy people [18]. ECG signals are influenced by the motion of thoracic cavity since electrodes are placed on chests [19]. In addition, ECG electrodes could be used to record the cardiac electric activity and motion of the chest at the same time. The baseline offset caused by patient's movement could cause a problem in detecting the $\mathrm{R}$ peak of the $\mathrm{QRS}$ complex. For this reason, clearing the signal would involve discarding the A9 wavelet coefficient (low frequency components) and applying a band-pass filter with cutoff frequencies of $0.5-40 \mathrm{~Hz}$. The modified PamTompkin method was utilized to find the R-peak, which was used to compute the R-R intervals. We utilized statistical time-domain features such as mean, variance, kurtosis and R-R interval duration, and spectral features such as spectral flatness, spectral centroid, spectral spread, spectral decrease and spectral slope. On the other hand, we extracted means of Power Spectrum Density in $10-20 \mathrm{~Hz}$ (mean of $P S D_{10 / 20}$ ) and $80-100 \mathrm{~Hz}$ (mean of $P S D_{80 / 100}$ ) respectively, since the lower heart rate results in fewer ECG cycles and less typical saw-tooth morphology and typical respiratory overexertion increases the respiratory rate.

\section{B. EEG features}

It has been shown that a decrease in Delta wave $(1-4 \mathrm{~Hz})$ is accompanied by an increase in Theta $(4-8 \mathrm{~Hz})$ and Alpha waves $(8-13 \mathrm{~Hz})$ [20]. We utilized wavelet transformation to decompose EEG window signals and obtain approximated Delta, Theta and Alpha waves. We used level-8 wavelet transformation and Daubechies wavelets 2. Since the sample rate is $200 \mathrm{~Hz}$, D8 and D7 $(0.78125-3.125 \mathrm{~Hz})$ were considered as the Delta wave, D6 $(3.125-6.25 \mathrm{~Hz})$ was considered as Theta wave and D5 $(6.25-12.5 \mathrm{~Hz})$ was considered as the Alpha wave. The A9 wavelet coefficient was removed and a band-pass filter with cut-off frequencies of $0.5-45 \mathrm{~Hz}$ was applied to remove noise. The EEG feature set included: mean of absolute value, maximum, minimum, standard deviations and variance of EEG windows of Delta, Theta and Alpha.

\section{EMG features}

There are bursts of EMG activity in apnea periods [15], and they cause changes in the frequency domain. In addition, 
bursts result in saw-tooth morphology, which causes higher values in low-frequency band. More chin activity causes an increase in high-frequency band. Power spectrum density of the EMG signal was first obtained, and then the maximum, mean, summation and median for each windows were calculated. More specifically, the frequency associated with the maximum value (freq - max of PSD) and the summation within $80-100 \mathrm{~Hz}$ (sum of $P S D_{80 / 100}$ ) were obtained. The decomposition of a window was performed using the wavelet transform, and for this work, it was determined to include the variance and standard deviation of D1 in the feature set.

\section{Oxygen saturation features}

One of the most important apnea criteria is the severity and duration of oxygen desaturation, i.e., the drop of oxygen level below a certain threshold (usually $3 \%$ or $4 \%$ decline from baseline) [21]. Sleep apnea events are usually associated with pseudo-periodic patterns [22], [23]. Sudden downturns and relatively sudden recoveries in oximetry saturation are linked to apnea, and these sudden changes show some typical saw-tooth morphology in the oximetry curve [21]. Firstly, $\mathrm{SaO} 2$ signals were down-sampled from $200 \mathrm{~Hz}$ to $1 \mathrm{~Hz}$. $\mathrm{SaO} 2$ feature set includes medians of windows. In addition, rapid restoration events defined as an increase in $\mathrm{SaO} 2>4 \%$ within a time interval of $10 \mathrm{~s}\left(O D I_{4-10}\right)$ were identified, and the mean of Power spectral density within the frequency range of 0.016 and $0.05 \mathrm{~Hz}$ (mean of $P S D_{.016 / .05}$ ) was added to the feature set, since cyclic oxygen desaturation shows a peak at low-frequency range in Power spectral density. Also, the standard deviation called $S D_{1}$ was computed, and this parameter indicates the short-term variability of $\mathrm{SaO} 2$ signal [21].

\section{E. Airflow features}

An apnea criterion is defined as a decrease in the airflow to less than $10 \%$ from its basal value for at least 10s [5]. Also, when a patient misses two breaths, then this would indicate an occurrence of apnea [24]. Firstly, recordings were down-sampled from $200 \mathrm{~Hz}$ to $1 \mathrm{~Hz}$. Time-domain statistical features included the mean, median, and standard deviation. Power spectral density within the frequency ranges of 0 and $0.1 \mathrm{~Hz}$ (mean of $P S D_{.0 / .1}$ ) and 0.4 and $0.5 \mathrm{~Hz}$ (mean of $P S D_{.4 / .5}$ ) were calculated, since missing two cycles causes less respiratory frequency range and fast respiratory overexertion increases the respiratory frequency. Wavelet transformation, with depth 3 and Daubechies wavelets 3, was utilized. Means of every reconstruction detail levels (D3, D2, and D1) and the approximation level (A3) were computed.

\section{F. Abdominal and thoracic features}

During apnea periods, the respiratory muscles become active, which means movements of patients thorax and abdomen [8]. Firstly, feature sets were computed using abdominal signals. The time-domain feature set included: summation, standard deviation, mean of the absolute value of every window. Power spectrum density was used to obtain the mean of the frequency range from 80 to $100 \mathrm{~Hz}$ (mean of
$P S D_{80 / 100}$ ) and means of D1 and D2 were computed. Then other features were also extracted from the thoracic signals, which include summation, median, standard deviation, mean and variance of each window. The mean was extracted in the spectral band (80 to $100 \mathrm{~Hz}$ ) (mean of $P S D_{80 / 100}$ ).

\section{RESULTS}

Feature selection is an important step before the classification stage since it can optimize classification performance. A small subset with high-discriminatory power is obtained by feature selection to avoid the curse of dimensionality problem and reduce the complexity of the classification. Statistical significance of the features was analyzed. Oneway analysis of variance (ANOVA) and Rank-Sum test were used for descriptive comparison of features between apnea and normal since these two test methods assess for a given feature the difference between the two groups. ANOVA is used to analyze the differences among group means and associated labels, and the p-value was calculated. A p-value that is $<0.05$ indicates significant difference between means of the subgroups. Rank-sum test is a non-parametric test and a randomly selected value from the first group is compared with a randomly selected one from the second group. This test is used to confirm two groups are independent. The pvalue is also computed for rank-sum test. A p-value of 1 indicates that the two groups are independent.

All individual features were evaluated for two groups (apnea and normal) to show the statistically significance, as shown in Figure 2. Results were coherent for the time and frequency domain. In the time domain, ECG features of variance, kurtoses and RR intervals were found to quantify apneas, which agreed with slower heart rate for SA patients that is reported in literature. For the airflow features, means, medians and standard deviations of windows were different between apnea and normal, due to some airflow decrease and recovering of normal breaths. In EEG signals, means of absolute windows, maximum, minimum, standard deviation and variance of windows represented events triggering an awakening activity, and they were different between apnea and normal. Due to the declined in $\mathrm{SaO} 2$ parameters during apnea, medians of windows, $O D I_{4-10}$ and $S D_{1}$ were found relevant (this agrees with reported studies). For features extracted from abdominal signals, summation, standard deviation, and mean of windows were found to partially agree with the reported studies, which mentioned that the respiratory muscles are active, however, the obtained $\mathrm{p}$ of ANOVA was $<0.05$ but $\mathrm{p}$ of Rank-Sum was 0 . Meanwhile, summation, median, mean and variance in thoracic signals were found relevant to the classification of apneas.

In frequency-domain statistics, the following ECG features were found relevant: spectral flatness, spectral centroid, spectral spread, spectral decrease, spectral slope, mean of $P S D_{10 / 20}$ and mean of $P S D_{80 / 100}$. For airflow features, mean of $P S D_{.0 / .1}$, mean of $P S D_{.4 / .5}$, mean of A3 were able to differentiate between apnea and normal. In EEG signals, maximums of Alpha and Theta waves, minimums 
Fig. 2. Statistical significance of preselected features from different signals (yellow features are time-domain)

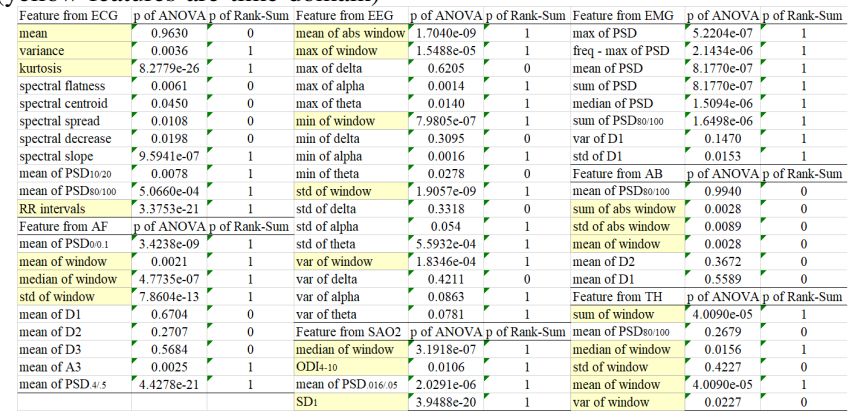

of Alpha and Theta waves, standard deviations of Alpha and Theta waves and variances of Alpha and Theta waves agreed with reported studies, as they identified decrease in Delta wave and increase in Alpha and Theta waves. Due to sudden downturns and fast recoveries in $\mathrm{SaO} 2$ that occur during apnea, mean of $P S D_{.016 / .05}$ was found relevant. About EMG features, maximum, mean, summation and median of PSD, freq - max of PSD, summation of $P S D_{80 / 100}$, variance, and standard deviation of D1 were all found to be relevant features, as they reflected bursts of EMG activity in apneas. Similarly, oxygen saturation features were also found to be linked to physiological criteria. During apnea, the decline from baseline is widely defined to confirm sudden downturns that are followed by sudden recoveries, which both lead to changes in the frequency domain.

\section{CONCLUSION}

In this paper, we have presented a new algorithm to extract and evaluate feature sets from multiple PSG signals, which included ECG, EMG, EEG, airflow, SaO2, abdominal and thoracic signals. From these signals, time- and frequencydomain features were used to reflect changes in behavior between apnea and normal PSG. Since the relevant features improve classification accuracy, ANOVA and Rank-Sum test were utilized to analyze and quantify the performance of features. The proposed approach proved useful in identifying features that provided the best contribution in separating the two classes of apnea and normal. These features could enhance the diagnosis of sleep apnea when fed to a classification algorithm.

\section{REFERENCES}

[1] W. Flemons, D. Buysse, S. Redline, A. Oack, K. Strohl, J. Wheatley, T. Young, N. Douglas, P. Levy, W. McNicolas, et al., "Sleep-related breathing disorders in adults: recommendations for syndrome definition and measurement techniques in clinical research," Sleep, vol. 22, no. 5, pp. 667-689, 1999.

[2] J. Chan, J. C. Edman, and P. J. Koltai, "Obstructive sleep apnea in children," American family physician, vol. 69, no. 5, pp. 1147-1160, 2004.

[3] N. Pombo, N. Garcia, and K. Bousson, "Classification techniques on computerized systems to predict and/or to detect apnea: A systematic review," Computer Methods and Programs in Biomedicine, 2017.

[4] J. V. Marcos, R. Hornero, D. Álvarez, F. del Campo, C. Zamarrón, and M. López, "Utility of multilayer perceptron neural network classifiers in the diagnosis of the obstructive sleep apnoea syndrome from nocturnal oximetry," computer methods and programs in biomedicine, vol. 92, no. 1, pp. 79-89, 2008.
[5] A. Otero, P. Félix, S. Barro, and C. Zamarrón, "A structural knowledge-based proposal for the identification and characterization of apnoea episodes," Applied Soft Computing, vol. 12, no. 1, pp. 516526, 2012.

[6] S. Güneş, K. Polat, and Ş. Yosunkaya, "Multi-class f-score feature selection approach to classification of obstructive sleep apnea syndrome," Expert systems with applications, vol. 37, no. 2, pp. 998-1004, 2010

[7] O. Fontenla-Romero, B. Guijarro-Berdiñas, A. Alonso-Betanzos, and V. Moret-Bonillo, "A new method for sleep apnea classification using wavelets and feedforward neural networks," Artificial Intelligence in Medicine, vol. 34, no. 1, pp. 65-76, 2005.

[8] A. Otero, P. Félix, and M. R. Álvarez, "Algorithms for the analysis of polysomnographic recordings with customizable criteria," Expert Systems with Applications, vol. 38, no. 8, pp. 10133-10146, 2011.

[9] J. D. Martínez-Vargas, L. M. Sepúlveda-Cano, C. Travieso-Gonzalez, and G. Castellanos-Dominguez, "Detection of obstructive sleep apnoea using dynamic filter-banked features," Expert Systems with Applications, vol. 39, no. 10, pp. 9118-9128, 2012.

[10] A. R. Hassan and M. A. Haque, "Computer-aided obstructive sleep apnea screening from single-lead electrocardiogram using statistical and spectral features and bootstrap aggregating," Biocybernetics and Biomedical Engineering, vol. 36, no. 1, pp. 256-266, 2016.

[11] C.-C. Hsu and P.-T. Shih, "A novel sleep apnea detection system in electroencephalogram using frequency variation," Expert Systems with Applications, vol. 38, no. 5, pp. 6014-6024, 2011.

[12] E. D. Übeyli, D. Cvetkovic, G. Holland, and I. Cosic, "Adaptive neurofuzzy inference system employing wavelet coefficients for detection of alterations in sleep eeg activity during hypopnoea episodes," Digital Signal Processing, vol. 20, no. 3, pp. 678-691, 2010.

[13] J. Huang, J. Zhang, S. P. Lam, S. X. Li, C. K. W. Ho, V. Lam, M. W. M Yu, and Y.-K. Wing, "Amelioration of obstructive sleep apnea in rem sleep behavior disorder: implications for the neuromuscular control of osa," Sleep, vol. 34, no. 7, pp. 909-915, 2011.

[14] R. Ferri, J.-F. Gagnon, R. B. Postuma, F. Rundo, and J. Y. Montplaisir, "Comparison between an automatic and a visual scoring method of the chin muscle tone during rapid eye movement sleep," Sleep medicine, vol. 15, no. 6, pp. 661-665, 2014.

[15] J. W. Burns, F. B. Consens, R. J. Little, K. J. Angell, S. Gilman, and R. D. Chervin, "Emg variance during polysomnography as an assessment for rem sleep behavior disorder," Sleep, vol. 30, no. 12 , pp. 1771-1778, 2007.

[16] N. Sezgin and M. E. Tagluk, "Energy based feature extraction for classification of sleep apnea syndrome," Computers in biology and medicine, vol. 39, no. 11, pp. 1043-1050, 2009.

[17] M. E. Tagluk, M. Akin, and N. Sezgin, "Classıfıcation of sleep apnea by using wavelet transform and artificial neural networks," Expert Systems with Applications, vol. 37, no. 2, pp. 1600-1607, 2010.

[18] G. Aljadeff, D. Gozal, V. L. Schechtman, B. Burrell, R. M. Harper, and S. L. Davidson Ward, "Heart rate variability in children with obstructive sleep apnea," Sleep, vol. 20, no. 2, pp. 151-157, 1997.

[19] A. Travaglini, C. Lamberti, J. DeBie, and M. Ferri, "Respiratory signal derived from eight-lead ecg," in Computers in Cardiology 1998, pp. 65-68, IEEE, 1998.

[20] P. Xavier, K. Behbehani, D. Watenpaugh, and J. R. Burk, "Detecting electroencephalography variations due to sleep disordered breathing events," in Engineering in Medicine and Biology Society, 2007. EMBS 2007. 29th Annual International Conference of the IEEE, pp. 6097 6100, IEEE, 2007.

[21] D. S. Morillo, J. L. Rojas, L. F. Crespo, A. León, and N. Gross, "Poincaré analysis of an overnight arterial oxygen saturation signal applied to the diagnosis of sleep apnea hypopnea syndrome," Physiological measurement, vol. 30, no. 4, p. 405, 2009

[22] D. S. Morillo, N. Gross, A. León, and L. F. Crespo, "Automated frequency domain analysis of oxygen saturation as a screening tool for sahs," Medical engineering \& physics, vol. 34, no. 7, pp. 946953, 2012.

[23] A. Garde, P. Dehkordi, W. Karlen, D. Wensley, J. M. Ansermino, and G. A. Dumont, "Development of a screening tool for sleep disordered breathing in children using the phone oximeter," PloS one, vol. 9, no. 11, p. e112959, 2014.

[24] G. C. Gutiérrez-Tobal, M. L. Alonso-Álvarez, D. Álvarez, F. del Campo, J. Terán-Santos, and R. Hornero, "Diagnosis of pediatric obstructive sleep apnea: Preliminary findings using automatic analysis of airflow and oximetry recordings obtained at patients home," Biomedical Signal Processing and Control, vol. 18, pp. 401-407, 2015. 